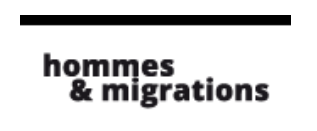

Hommes \& migrations

Revue française de référence sur les dynamiques

migratoires

$1290 \mid 2011$

Travailleurs sociaux et migrations

\title{
L'intervention sociale et la question ethnique
}

Entre ethnicisation, déprofessionnalisation et pacification

\section{Manuel Boucher et Mohamed Belqasmi}

\section{OpenEdition}

1 Journals

\section{Édition électronique}

URL : http://journals.openedition.org/hommesmigrations/731

DOI : 10.4000/hommesmigrations.731

ISSN : 2262-3353

Éditeur

Musée national de l'histoire de l'immigration

Édition imprimée

Date de publication : 1 mars 2011

Pagination : 22-32

ISSN : 1142-852X

\section{Référence électronique}

Manuel Boucher et Mohamed Belqasmi, «L'intervention sociale et la question ethnique », Hommes \& migrations [En ligne], 1290 | 2011, mis en ligne le 31 décembre 2013, consulté le 22 avril 2019. URL : http://journals.openedition.org/hommesmigrations/731; DOI : 10.4000/hommesmigrations.731

Tous droits réservés 


\section{L'intervention sociale et la question ethnique}

\section{Entre ethnicisation, déprofessionnalisation et pacification}

Par Manuel Boucher, sociologue, directeur scientifique (HDR) du Laboratoire d'étude et de recherche sociales (LERS) de l'Institut du développement social (IDS-IRTS) de Haute-Normandie - Canteleu/Rouen et membre associé du CADIS-EHESS, président de l'Association des chercheurs des organismes de la formation et de l'intervention sociales (ACOFIS),

et Mohamed Belqasmi, sociologue, attaché de recherche au LERS-IDS, doctorant à l'université Nice-Sophia Antipolis-URMIS, secrétaire de l'ACOFIS

Les professions sociales cristallisent les tensions internes de la société dans laquelle elles interviennent. Dans la société française

contemporaine, la dimension ethnique des rapports sociaux tend à prendre de l'importance. Dans ce contexte, le recrutement des acteurs sociaux dans les quartiers populaires s'appuie de plus en plus sur leur origine au détriment de leur qualification. Cette mise en avant de leurs compétences ethnoculturelles laisse entrevoir les processus d'assignation identitaire qui surdéterminent leur action. 
Au sein des transformations de modes de prise en charge sociale des difficultés des populations des quartiers populaires, notamment des migrants et de leurs descendants, nous assistons au développement et au renforcement d'un système de pacification sociale "indigène" dont le ressort principal est l'autochtonie d'intervenants sociaux précarisés. En pleine mutation, l'espace du travail social est en effet, à l'instar d'autres secteurs professionnels, producteur de processus de "racisation" et de discriminations ethno-raciales. Dans une dynamique de hiérarchisation des différents segments qui composent cet espace professionnel polymorphe, parallèlement au développement de nouveaux emplois destinés à encadrer les populations "turbulentes" des quartiers populaires, on observe des situations d'inégalité et de différenciation des intervenants sociaux basées sur leur "origine" réelle ou supposée. Ils font face à l'injonction de mobiliser des "compétences ethniques" pour intervenir auprès des populations au détriment de leur reconnaissance sociale et professionnelle ${ }^{(1)}$.

\section{Un espace professionnel en recomposition}

Les professions sociales se présentent aujourd'hui comme un véritable archipel dont le noyau historique s'est constitué grâce à une logique de qualification et de définition de titres, de statuts et d'emplois, cependant une recomposition des emplois est en cours depuis une quinzaine d'années. Il existe aujourd'hui principalement deux types de "marchés du travail". Le premier, relativement fermé, est tendanciellement réservé aux diplômés du travail social. Il concerne le "noyau dur" des professions canoniques, exerçant essentiellement dans les secteurs du handicap, de la protection de l'enfance ou des personnes et familles en difficulté. Ce secteur privilégie la logique de qualification collective. Le second secteur de l'intervention sociale est plus ouvert aux recrutements externes. Il concerne des sous-secteurs en expansion (aide à domicile) ou en émergence (médiation urbaine, insertion, développement local, accompagnement des immigrés, etc.). Ce "secteur périphérique" ne semble pas particulièrement enclin à favoriser la professionnalisation de ses intervenants sociaux pour de multiples raisons (financières, politiques, de méconnaissance du secteur social...). Les critères de recrutement privilégiés par les employeurs du "secteur classique" relèvent à la fois du "modèle de la qualification" et du "modèle de la compétence" : il s'agit d'associer la possession d'un diplôme du travail social, des compétences relationnelles et des capacités d'initiative et d'adaptation dans le cadre d'un travail d'équipe.

Dans le secteur périphérique, dans un contexte de difficultés financières accrues au sein des milieux associatifs, les employeurs privilégient l'expérience auprès de publics en difficulté et la proximité avec les usagers plutôt que la possession d'un diplôme 
canonique du travail social. C'est dans cette dynamique de dualisation relative de l'intervention sociale que l'on constate au sein de certains espaces urbains des phénomènes d'ethnicisation du travail social, qui se manifestent notamment par l'instrumentalisation d'intervenants sociaux "d'origine étrangère".

\section{Aspects méthodologiques}

L'enquête sur laquelle s'appuie cet article a porté sur les processus d'ethnicisation et de racisation du champ social, à partir de données principalement quantitatives récoltées dans la région de Haute-Normandie au courant de l'année 2006. Nous avons cherché à savoir si du côté du système l'intervention sociale est engagée dans un processus d'ethnicisation favorisé par l'éclatement de ce secteur. Et si du côté des acteurs, les intervenants sociaux sont également les coproducteurs de l'ethnicisation et de la racisation des rapports sociaux (hétéro-racisation, auto-racisation).

Pour dresser un bref tableau du profil des répondants, on peut souligner qu'ils exercent en grande majorité dans le secteur classique, principalement dans des établissements sociaux, et pour un peu moins d'un tiers d'entre eux (31\%) dans le secteur périphérique, majoritairement dans des structures de l'intervention sociale urbaine et de l'insertion. Pour la plupart, ces professionnels sociaux sont des enfants d'ouvriers (38 \%) et ont une fois sur dix (12 \%) un père né en Afrique du Nord ou subsaharienne. On peut aussi noter que par rapport aux intervenants sociaux exerçant dans le secteur classique, ceux du secteur périphérique possèdent certaines caractéristiques : ils sont respectivement plus souvent issus des couches populaires ( $42 \%$ ont un père ouvrier, contre 35,3\%), plus précaires du point de vue de l'emploi (43,5\% sont en CDD ou en contrat aidé, contre $24,3 \%$ ), moins qualifiés $(28,5 \%$ ne détiennent pas de qualification professionnelle du champ social, contre 6,7\%) et plus fréquemment "d'origine étrangère" (22,3 \% ont un père né en Afrique du Nord ou subsaharienne, contre 7,7 \%).

\section{Des intervenants sociaux moins qualifiés et précarisés}

Les intervenants sociaux "d'origine africaine" sont non seulement surreprésentés dans le secteur périphérique mais ils y sont aussi largement concentrés : la majorité de ces personnes est regroupée dans ce secteur $(55,7 \%$ ), contre seulement le quart de celles "d'origine métropolitaine" (26,4 \%).La forte concentration de ces professionnels issus, pour le dire rapidement, des migrations postcoloniales dans les segments les plus 
précaires de l'espace de l'intervention sociale a dès lors des répercussions sur leur accès à la qualification et à un statut salarial sécurisé. Ainsi, du point de vue de l'accès à la qualification professionnelle, les intervenants sociaux "d'origine africaine" sont beaucoup moins souvent détenteurs d'un titre professionnel du travail social et de l'animation que leurs homologues dont le père est né en France métropolitaine. Même s'ils sont peu nombreux, on peut observer que près de la moitié des intervenants sociaux "d'origine subsaharienne" (45,4 \%) ne détiennent pas de diplôme professionnel, contre près du tiers de ceux "d'origine nord-africaine" (30,7 \%) et seulement 11,1\% de ceux "d'origine métropolitaine". Par ailleurs, concernant la stabilité des statuts d'emploi, les intervenants sociaux "d'origine africaine" bénéficient globalement de contrats plus précaires : 34,9\% d'entre eux sont en contrat à durée déterminée (CDD) ou en contrat aidé, contre 29,5\% de ceux "d'origine métropolitaine". Ces situations d'inégalités et de "concentration ethnique" sont accentuées dans les milieux de l'intervention sociale urbaine connus pour leur déficit de professionnalisation comme, par exemple, les structures de médiation sociale où l'on retrouve une majorité de personnels sociaux "d'origine étrangère" (sur 10 répondants enquêtés exerçant dans ce type de structure, 4 sont "d'origine africaine", 6 "d'origine non européenne").

Ces métiers de la médiation de proximité répondent aux objectifs de la décentralisation, de la territorialisation mais aussi de la régulation sociale par des actions positives de traitement social du chômage ${ }^{(2)}$. En revanche, les modes de régulation ethnique opérés dans les quartiers populaires sont diversement appréciés. D'un côté, ils sont critiqués comme des modes de pacification sociale, de l'autre côté, ils sont considérés comme un moyen de remobiliser et de resocialiser des jeunes rebelles stigmatisés comme des figures de dangerosité.

\section{Le travail social à l'épreuve de l'ethnification}

Dans tous les cas, notre enquête souligne qu'il existe au sein de l'espace de l'intervention sociale une spécialisation sectorielle des professionnels "d'origine africaine". Cette spécialisation peut être interprétée comme un système d'aiguillage de populations en quête d'insertion qui, par vocation ou par défaut, s'orientent vers des structures plus accessibles parce qu'elles n'attirent que peu de professionnels "canoniques" qualifiés. Le champ de l'intervention sociale constitue en effet un marché de l'emploi relativement tendu pour de nombreuses raisons : la pénurie de personnels qualifiés par rapport aux besoins, l'effet de la pyramide des âges et les nombreux départs en retraite qui en découlent, etc. Les cadres d'intervention considérés comme difficiles, 
contraignants ou mal rémunérés par les plus qualifiés se trouvent ainsi délaissés. Il est certain que la détention d'une qualification professionnelle reconnue permet de choisir son lieu de travail, donc de laisser vacants les emplois les moins attractifs, comme l'indique le responsable d'un centre éducatif renforcé (CER) spécialisé dans l'accueil des jeunes dits "difficiles" interrogé durant l'enquête : "La majorité des personnes qui se présentent à des entretiens d'embauche au CER sont issues de l'immigration ou de quartiers populaires. En fait, les embauches que l'on fait, la plupart du temps, se font de bouche à oreille. Nous avons une population difficile. Et on n'a pas une grande

On peut se demander si la qualification professionnelle, ou plutôt son absence, constitue le seul critère d'embauche (par défaut) des intervenants sociaux "d"origine africaine". demande de la part des éducateurs formés pour travailler en CER."

Or on peut se demander si la qualification professionnelle, ou plutôt son absence, constitue le seul critère d'embauche (par défaut) des intervenants sociaux "d'origine africaine" dans le secteur périphérique ou dans des sous-secteurs du champ social classique considérés comme particulièrement éprouvants. D'autres types d'indicateurs de compétences sont-ils pris en compte par les employeurs pour décider de l'embauche d'un postulant "d'origine africaine" ? Afin de répondre à cette question, nous avons demandé aux enquêtés quels étaient selon eux les principaux critères qui avaient été retenus par leur employeur pour les embaucher.

\section{Une valorisation pragmatique des compétences ethnoculturelles}

La concentration des professionnels "d'origine africaine" dans les champs d'intervention les moins exigeants du point de vue de la détention d'un diplôme professionnel et les plus précaires du point de vue salarial pose la question des autres critères qui président à leur embauche. Si la qualification professionnelle n'est pas un critère déterminant d'embauche des intervenants sociaux "d'origine africaine", en particulier dans le secteur périphérique, l'attribution de qualités référées à leur parcours de vie parait jouer en leur faveur et ils en sont d'ailleurs tout à fait conscients. Ainsi, 37,1 \% des intervenants sociaux "d'origine africaine" ont le sentiment d'avoir été recrutés pour leur "expérience de vie dans un quartier populaire", contre 9,7 \% pour ceux "d'origine métropolitaine". De plus, 35,5\% des intervenants sociaux "d'origine africaine" disent avoir été embauchés grâce à leur "proximité avec les publics et les 
communautés en présence", contre 10,2 \% pour ceux “d'origine métropolitaine”. Ces phénomènes de concentration sont également perceptibles à l'intérieur des différents secteurs : plus de 90 \% des personnels sociaux exerçant en Maison d'enfants à caractère social (MECS) sont "d'origine européenne", alors que sur 10 professionnels travaillant en Centre éducatif fermé (CEF), 4 sont "d'origine africaine". Le directeur d'un CEF interrogé a souligné que 80 \% des personnes qui postulent pour assumer une fonction éducative dans son établissement sont d'origine étrangère, notamment du Maghreb et d'Afrique noire.

On pourrait se contenter de constater la reconnaissance, implicite ou explicite, de la "différence culturelle" par certains employeurs et l'interpréter comme le simple signe de l'évolution des pratiques de recrutement prenant dorénavant en compte des compétences humaines ${ }^{(3)}$ particulières, notamment des "compétences ethnoculturelles". Dans la pratique, on s'aperçoit que c'est notamment parce qu'ils sont censés intervenir auprès de populations elles-mêmes majoritairement "d'origine étrangère" que ces professionnels sont en partie embauchés. Dans la mesure où ces acteurs "d'origine étrangère" interviennent en grande partie auprès de populations qui le sont elles aussi (ou sont perçues comme telles), ils se trouvent mécaniquement dans des situations qui les mènent à mobiliser dans leurs actions quotidiennes leur "origine sociale et culturelle", voire parfois leur "couleur de peau" lorsqu'ils sont "d'origine subsaharienne". Ainsi, les personnels sociaux "d'origine africaine" disent faire appel à leur "origine sociale ou culturelle" pour intervenir dans 32,2\% des cas, contre seulement $6 \%$ de ceux "d'origine métropolitaine". Par ailleurs, sur 23 personnes "d'origine subsaharienne", 3 affirment quand même mobiliser leur "couleur de peau" dans leurs interventions.

\section{Le danger de l'assignation identitaire}

La prise en compte par des employeurs de la proximité socioculturelle (supposée) avec leurs futurs usagers des postulants à un poste d'intervenant social peut paraître motivée par une démarche pragmatique d'intervention auprès de populations "d'origine étrangère" : ils auraient en somme plus de facilités à intervenir auprès de leurs semblables, ils seraient réputés "savoir y faire". Les "compétences ethniques" sont ainsi des critères de recrutement officieux et euphémisés (connaissance du public et de ses spécificités, rapports avec les familles et les “communautés en présence”...).En effet, les intervenants sociaux "culturalisés" et "phénotypés"(4)" sont considérés comme directement opérationnels pour faire du contrôle social avec les jeunes des quartiers populaires et les familles immigrées, comme l'illustre cet extrait d'entretien réalisé 
avec un responsable de médiateurs sociaux employés par une entreprise de transports en commun : "J'ai vu un agent de médiation intervenir en arabe pour remettre en place un jeune Arabe qui insultait en arabe une conductrice de bus. Des médiateurs disent : 'Si tu étais au bled, tu n'agirais pas comme ça, alors pourquoi tu le fais ici?' Ils essayent de faire ressortir les racines et cette notion de respect qui existe là-bas et qui n'existe pas ici."

Cette forme de division ethnique du travail social traduit également une forme d'accommodation de l'intervention sociale vis-à-vis de l'accroissement de l'ethnicisation des rapports sociaux dans la société française contemporaine encore plus développée dans les quartiers de ségrégation ${ }^{(5)}$. Ces pratiques font coïncider des formes d'ethnification des intervenants sociaux "d'origine étrangère" avec des pratiques de gestion de populations elles-mêmes souvent racisées ${ }^{(6)}$. Dans cette perspective, la prise en compte de "compétences ethnoculturelles" a beaucoup de chances de conduire à des formes d'assignation identitaire si elle n'est pas contrebalancée par des perspectives de reconnaissance professionnelle, notamment par l'accès à la qualification.

\section{Des ressources pacificatrices valorisées au sein d'un espace hiérarchisé}

Ces quelques données tendent à confirmer l'idée que coexistent deux modèles d'intervention sociale hiérarchiquement et politiquement inégaux. Au sein du secteur classique du travail social qui concerne le noyau dur des professions sociales (handicap, protection de l'enfance, réadaptation sociale), nous assistons à la recomposition d'une politique sociale complexe (État et Conseils généraux), héritée de l'après-guerre (Étatprovidence) et fondée sur les valeurs égalitaristes républicaines. Elle est chargée, d'une part, de traiter les dysfonctionnements sociaux (aide sociale, handicap, enfance en danger, traitement de la délinquance....) et, d'autre part, de combattre institutionnellement, politiquement et économiquement les effets néfastes d'une société capitaliste inégalitaire (traitement social du chômage, politique du logement social, lutte contre les discriminations). Au sein du secteur périphérique qui concerne notamment des activités en émergence (la médiation urbaine, l'insertion, le développement local, l'accompagnement des immigrés), nous constatons l'expansion d'une politique de pacification sociale hétérogène et pragmatique commandée essentiellement par des élus locaux. Sa fonction est principalement de maintenir l'ordre dans des espaces de ségrégation en développant des logiques coercitives et de médiation communautaire (ethnique, religieuse, morale) assumées notamment par des acteurs ethnicisés instrumentalisés par les pouvoirs publics, cherchant le plus souvent une opportunité d'insertion sociale mais aussi parfois du pouvoir politique. 
Contrairement à ce que l'on pourrait penser, à l'instar d'autres champs d'activités plus classiquement soupçonnés de racisme comme la police ${ }^{(7)}$, le champ de l'intervention sociale est lui aussi producteur de processus de "racisation" et de discriminations ethno-raciales. Nous assistons à un phénomène d'ethnicisation, voire de racisation d'intervenants sociaux "d'origine étrangère" ou considérés comme tels, corrélativement à un processus de dualisation de l'intervention sociale. Même si le modèle de la compétence n'a pas complètement remplacé le modèle de la qualification dans des secteurs traditionnels (l'inadaptation, le handicap, la protection de l'enfance, l'action

Lintervention sociale s'adapte en creux à la recomposition des modes de contrôle social dans un contexte d'installation de la précarité. sociale de secteur...), ce dernier n'est plus hégémonique, notamment dans le cadre de la politique de la ville. Au travailleur social "classique" au professionnalisme et à la déontologie affirmés, l'élu local préfère des intervenants sociaux ayant une culture professionnelle incertaine ou un statut précaire comme les agents locaux de médiation sociale, souvent des jeunes d'origine populaire, plus malléables, qui troquent une violence réelle ou supposée contre une promesse de promotion sociale via les métiers de l'animation et de la médiation urbaine ${ }^{(8)}$.

\section{La différence au service de la paix sociale}

Le travail social comme profession unifiée est donc remis en cause par le développement de "métiers" aux contours mal définis. Le cadre rationnel et déontologique du travail social régresse au profit d'une "légitimité émotionnelle" en lien avec une conception pratique et territoriale de l'action sociale. En effet, dans un contexte d'augmentation des phénomènes d'insécurité sociale et civile ${ }^{(9)}$ mettant potentiellement en péril la cohésion sociale, les populations des quartiers défavorisés, notamment les jeunes racisés, apparaissent soit comme des fauteurs de troubles, soit comme des potentiels pacificateurs sociaux (médiateurs, prédicateurs sociaux, agents d'ambiance...) chargés de canaliser les premiers ${ }^{(10)}$. Pour les pouvoirs publics - l'État et les collectivités territoriales -, il s'agit de satisfaire à une multitude de besoins et demandes non plus nécessairement rattachés à des métiers du social déjà connus. Pour tous ces représentants de l'autorité publique, il s'agit avant tout de répondre efficacement à la précarité, à l'exclusion, à la violence de proximité. On constate alors une "hiérarchisation" de l'intervention sociale. Les nouveaux intervenants sociaux sont recrutés selon deux critères principaux : une logique 
de recrutement sociologiquement ciblée à travers des critères générationnels, ethniques ou de territoire ; une logique de spécialisation sectorielle et technique.

Dans ce contexte, ce qui compte au premier chef pour beaucoup d'employeurs, ce n'est pas la capacité des intervenants sociaux à analyser des "situations problèmes", mais leur capacité à contenir des désordres potentiels, comme le révèle cet extrait d'entretien avec le responsable éducatif d'une structure chargée de prendre en charge de jeunes délinquants : "Ici, on recrute des gens qui savent prendre en charge des jeunes difficiles. On ne les prend pas pour leur permettre de s'insérer, on les prend d'abord parce qu'ils sont bons dans le quotidien. J'ai pris un éducateur spécialisé, mais il a tenu huit jours. Dès qu'il était en relation interpersonnelle avec un gamin qui n'utilisait pas un bon langage, il ne supportait pas. Certes, il apportait quelque chose en termes d'analyse, mais il handicapait tout le monde. Ici, on demande aux éducs de faire avec le jeune."

On assiste dès lors à une dissolution relative des références déontologiques et des pratiques émancipatrices des intervenants sociaux au profit de l'utilisation de ressources pacificatrices ${ }^{(11)}$. Dans ce contexte, la “différence culturelle ${ }^{(12) "}$ " n'est pas reconnue comme une dimension importante de l'action sociale professionnelle contemporaine mais elle est essentiellement instrumentalisée pour assurer le maintien de l'ordre.

\section{Les contradictions de la division ethnique}

L'essentialisation des intervenants sociaux principalement recrutés selon des critères socio-ethniques peut amener ces acteurs à opérer une forme de contre-stigmatisation en transformant cet étiquetage en emblème de la différence culturelle.

La "catégorisation ethnique" des intervenants sociaux d'origine étrangère ou "phénotypés" produit un cercle vicieux : l'ethnicisation des acteurs sociaux conduit au renforcement de ce processus par les acteurs eux-mêmes cherchant à se distinguer. Ce processus a été extrêmement bien décrit par Norbert Elias et John L. Scotson dans leur monographie d'une petite ville d'Angleterre (Winston Parva) à propos de "the Established and the Outsiders ${ }^{(13)}$ ". Face à la stigmatisation dont ils sont victimes de la part des "enfants respectables" de la ville, "la minorité plus rebelle des jeunes gens se sentait rejetée, ceux-ci cherchaient à prendre leur revanche en s'appliquant à se conduire mal". Ces jeunes construisent un "renversement du stigmate" par une exagération des faits qu'on leur reproche (tapages, déprédations...). Les "ragots de rejet" deviennent ainsi des armes caduques car ces jeunes aspirent au rejet et aiment générer la contrariété. Ils aiment se définir comme la "minorité des pires" agissante. On peut néanmoins penser que le processus d'ethnicisation en cours dans l'intervention sociale donne une chance supplémentaire à des individus discriminés négativement, en raison de 
leur appartenance réelle ou supposée à des groupes socio-ethniques stigmatisés, de débuter une carrière professionnelle dans le champ social justement parce qu'ils mobilisent des ressources ethniques pour entrer dans l'emploi. Or, contrairement à cette idée, on peut également penser que les intervenants sociaux d'abord recrutés sur des bases ethno-raciales et territoriales pour surtout neutraliser les turbulences des nouvelles classes dangereuses ("jeunes de banlieue") entrent en fait dans une nasse. Leur entrée dans le secteur social en tant que "faisant fonction" peut en réalité servir d'alibi au déni de mobilisation institutionnelle pour que ces intervenants sociaux puissent entamer une carrière professionnelle nécessairement reliée à l'accès à une qualification reconnue au-delà du seul secteur périphérique qui les emploie majoritairement. Pour les intervenants sociaux hétéro-racisés, l'entrée dans le champ de l'intervention sociale peut en fait coïncider avec une promotion sociale par défaut.

\section{Conclusion}

De nombreux travaux sociologiques soulignent qu'au-delà des processus de stigmatisation territoriale des zones urbaines défavorisées, la marginalité des quartiers populaires est de plus en plus liée à leur ghettoïsation "ethno-raciale ${ }^{(14)}$ ". Les rapports de "race" organisent et divisent fréquemment la structure de la vie sociale. Les jeunes des cités opèrent régulièrement une lecture ethnique des rapports oppositionnels qu'ils entretiennent avec les institutions, notamment avec la police (bavures racistes) et l'école (ségrégation scolaire). Dans les quartiers, comme dans la société française, les relations sociales se sont peu à peu "racialisées". En fait, comme le soulignait déjà Didier Lapeyronnie ${ }^{(15)}$ au début des années quatre-vingt-dix, le problème n'est pas celui de l'intégration des immigrés et de leurs enfants, mais celui de groupes ethnifiés ou hétéro-racisés se caractérisant par leur confrontation quotidienne à de multiples formes de discriminations.

Quoi qu'il en soit, pour faire face au déficit de citoyenneté républicaine (liberté, égalité, fraternité, laïcité) vécu par toute une partie de la population disqualifiée et discriminée négativement ${ }^{(16)}$, notamment les personnes issues de l'immigration et/ou vivant dans les banlieues paupérisées, l'intervention sociale s'adapte en creux à la recomposition des modes de contrôle social dans un contexte d'installation de la précarité comme nouveau régime d'organisation du travail d'un pan entier du champ social. La production d'un processus d'ethnicisation/racisation et de déprofessionalisation surtout observé au sein du secteur périphérique - illustre cette adaptation. D'un point de vue idéal-typique, la mobilisation de compétences particulières, notamment ethnoraciales, pour produire de la pacification sociale conduit à essentialiser des intervenants 
sociaux ethnifiés, stigmatisés, désubjectivés et instrumentalisés. Pour légitimer leur utilité et opérer une contre-stigmatisation, ces acteurs sociaux sont dès lors contraints de mobiliser des caractéristiques "communautaires", voire raciales, renforçant ainsi le cercle vicieux de la déprofessionnalisation. De façon volontariste, les principaux acteurs du champ social (employeurs, syndicats de salariés, associations professionnelles, pouvoirs publics, organismes de formation) peuvent cependant rompre avec cette logique d'ethnicisation/déprofessionnalisation en affirmant un projet de professionnalisation pour tous les intervenants sociaux. Il s'agit dès lors de s'organiser pour permettre à l'ensemble des intervenants sociaux, qu' ils travaillent dans le secteur classique ou périphérique, de se construire comme des acteurs professionnels agissant à partir de références déontologiques plutôt qu'ethno-raciales. La reconnaissance de la différence culturelle des acteurs sociaux est une ressource uniquement lorsqu'elle peut s'articuler, d'un côté, avec l'affirmation d'une certaine distanciation/subjectivation, de l'autre, avec l'appropriation de valeurs universelles et professionnelles transmises notamment par la qualification, gage de l'autonomie des travailleurs sociaux.

\section{Notes}

1. Cet article reprend des éléments précédemment développés dans un ouvrage collectif sous la direction de Manuel Boucher intitulé Le Travail social face aux discriminations, Paris, éd. Aux lieux d'être, aujourd'hui épuisé.

2. Pierre Billon, "Les travailleurs sociaux dits 'issus de l'immigration'", in Informations sociales, $\mathrm{n}^{\circ}$ 113, 2004.

3. Robert L. Katz "Skills of an effective administrator", in Harvard Business Review, vol. 51, 1974.

4. Un phénotype sert à désigner l'ensemble des caractères individuels visibles liés à l'interaction du génotype et le l'environnement externe.

5. Éric Maurin, Le Ghetto français. Enquête sur le séparatisme social, Paris, Seuil, 2004.

6. Manuel Boucher, Turbulences. Comprendre les désordres urbains et leur régulation, Paris, éd. Aux lieux d'être, 2007 (réédition Téraèdre, 2010).

7. Sophie Body-Gendrot, Catherine Wihtol de Wenden, Police et discriminations raciales : le tabou français, Paris, éd. de l'Atelier, 2003.

8. Manuel Boucher, "La question ethnique, l'intervention sociale et la laïcité. Les enjeux des discriminations raciales dans le travail social", in Connexions, ${ }^{\circ}$ 83, 1, 2005, pp. 99-114.

9. Robert Castel, L'Insécurité sociale. Qu'est-ce qu'être protégé ?, Paris, Seuil, 2003.

10. Manuel Boucher, ALain Vulbeau (dir.)., Émergences culturelles et jeunesse populaire, Paris, L'Harmattan/INJEP, 2003.

11. Manuel Boucher, "Travail social, intervention sociale et pacification sociale. D'un projet d'intégration et de contrôle à une action d'émancipation ?", in Recherches sociologiques, vol. XXXV, n 3, 2004, pp. 137-151.

12. Michel Wieviorka, La Différence, Paris, Balland, 2001.

13. Norbet Elias N., John L. Scotson, Logiques de l'exclusion, Paris, Fayard, 1997.

14. Didier Lapeyronnie, Ghetto urbain, Paris, Robert Laffont, 2009 ; Manuel Boucher, Les Internés du ghetto.

Ethnographie des confrontations violentes dans une cité populaire, Paris, L'Harmattan, 2010.

15. Didier Lapeyronnie, L'Individu et les Minorités. La France et la Grande-Bretagne face à leurs immigrés, Paris, PUF, 1993.

16. Robert Castel, La Discrimination négative. Citoyens ou indigènes ?, Paris, Seuil, 2007. 